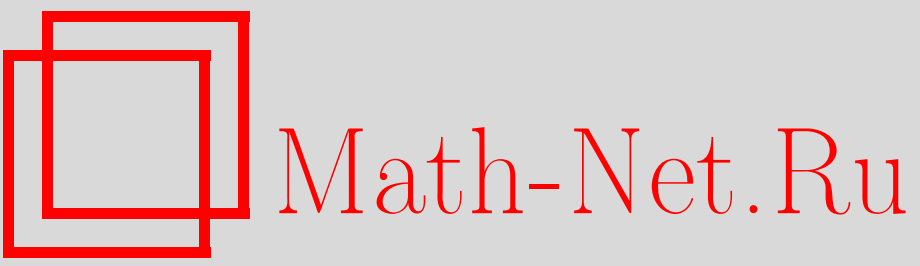

Л. В. Богачев, С. М. Зарбалиев, Предельные теоремы для одного класса случайных выпуклых ломаных, УМH, 1999, том 54, выпуск 4, 155-156

DOI: https://doi.org/10.4213/rm183

Использование Общероссийского математического портала Math-Net.Ru подразумевает, что вы прочитали и согласны с пользовательским соглашением

http://www.mathnet.ru/rus/agreement

Параметры загрузки:

IP: 3.95 .254 .165

26 апреля 2023 г., 16:22:49 


\title{
ПРЕДЕЛЬНЫЕ ТЕОРЕМЫ ДЛЯ ОДНОГО КЛАССА СЛУЧАЙНЫХ ВЫПУКЛЫХ ЛОМАНЫХ
}

\author{
Л.В. БОГАЧЕВ, С. М. ЗАРБАЛИЕВ
}

1. Введение. Мы будем рассматривать целочисленные выпуклые ломаные Г с вершинами в точках $\mathbb{Z}_{+}^{2}$, выходящие из начала координат и такие, что угол наклона каждого звена неотрицателен и не превосходит $\pi / 2$. Выпуклость означает, что наклон последовательных звеньев ломаной строго возрастает. Обозначим через $\mathscr{L}$ множество всех таких конечнозвенных ломаных, а через $\mathscr{L}_{n}$ - множество ломаных $\Gamma \in \mathscr{L}$ с фиксированным правым концом $n=\left(n_{1}, n_{2}\right)$.

Известно [1]-[3], что при $n_{1}=n_{2} \rightarrow \infty$ и скейлинге $\mathbb{Z}_{+}^{2} \mapsto n_{1}^{-1} \mathbb{Z}_{+}^{2}$ ансамбль $\mathscr{L}_{n}$ подчиняется закону больших чисел (относительно равномерного распределения вероятностей $P_{n}$ ), причем предельная кривая $\gamma_{0}$ задается уравнением $\sqrt{1-u_{1}}+\sqrt{u_{2}}=1$. Метод работы [3] основан на представлении меры $P_{n}$ как условного распределения, индуцированного на $\mathscr{L}_{n}$ подходящей мерой $Q$, заданной на $\mathscr{L}=\bigcup_{n} \mathscr{L}_{n}$. В настоящей заметке этот подход применяется для изучения статистических свойств $\mathscr{L}_{n}$ относительно одного нового класса распределений $P_{n}$, которые вводятся так, что $P_{n}(\Gamma)$ зависит от количества целых точек, лежащих на звеньях ломаной $\Gamma$.

2. Построение меры $P_{n}$. Положим $X:=\left\{\left(x_{1}, x_{2}\right) \in \mathbb{Z}_{+}^{2}:\right.$ н.о.д. $\left.\left(x_{1}, x_{2}\right)=1\right\}$, и пусть $\mathscr{F}$ - пространство функций $f: X \rightarrow \mathbb{Z}_{+}$. Заметим (см. [3]), что $\mathscr{L}$ эквивалентно множеству финитныц функций $\mathscr{F}_{0}:=\{f \in \mathscr{F}: \#(f(x)>0)<\infty\} ;$ при этом точке $x \in X$ c $f(x)>0$ отвечает звено, задаваемое вектором $\left(x_{1} f(x), x_{2} f(x)\right)$. (Функции $f(x) \equiv 0$ сопоставляется "тривиальная" ломаная с совпадающими концами.) Введем на $\mathscr{F}$ вероятностную меру $Q=Q z$ как распределение случайного поля $\nu=\nu(\cdot)$ на $X$ с независимыми значениями, причем

$$
Q_{z}\{\nu(x)=k\}:=g_{k} z^{k x} / G\left(z^{x}\right), \quad k=0,1,2, \ldots,
$$

где $\left\{g_{k}\right\}$ - положительная последовательность (не ограничивая общность, можно считать, что $\left.g_{0}=1\right), G(u):=\sum_{k=0}^{\infty} g_{k} u^{k}, u \in[0,1)$, - ее производящая функция, $z=\left(z_{1}, z_{2}\right)$, $0<z_{1}, z_{2}<1$, и $z^{k x}:=z_{1}^{k x_{1}} z_{2}^{k x_{2}}$. Корректность нормировки в (1) обеспечивается условием $\prod_{x \in X} G\left(z^{x}\right)<\infty$, откуда следует $\sum_{x \in X}\left(1-1 / G\left(z^{x}\right)\right)<\infty$ и, по лемме Бореля-Кантелли, $Q_{z}\left\{\nu(\cdot) \in \mathscr{F}_{0}\right\}=1$.

Будем далее рассматривать класс мер $\left\{Q_{z}\right\}$, который задается как выпуклая оболочка (симплекс Шоке), натянутая на семейство "крайних точек" $Q_{z}^{(r)}$ с $g_{k}=C_{k+r-1}^{k}=(r+k-1) \times$ $(r+k-2) \cdots r / k$ !, $0<r<\infty$. В частности, если $r=1$, то $g_{k} \equiv 1$ и случайная величина $\nu(x)$ имеет геометрическое распределение с параметром $q=z^{x}$ (ср. [3]). В общем случае $Q_{z}^{(r)}$ задает отрицательное биномиальное распределение $\nu(x)$ с параметрами $r$ и $q=z^{x}$ (см. [4; VI.8]).

Обозначим через $\xi_{i}:=\sum_{x} x_{i} \nu(x)(i=1,2)$ координаты правого конца ломаной $\Gamma$ и рассмотрим условное распределение

$$
P_{n}(\Gamma)=\frac{Q_{z}(\Gamma)}{Q_{z}\left\{\xi_{1}=n_{1}, \xi_{2}=n_{2}\right\}}, \quad \Gamma \in \mathscr{L}_{n}
$$

Из (1) вытекает, что $P_{n}$ не зависит от $z_{1}, z_{2}$; в частности, при $g_{k} \equiv 1$ распределение $P_{n}$ оказывается равномерным (ср. [3]). Подчиним $z_{1}, z_{2}$ условиям $\lim _{n \rightarrow \infty} n_{i}^{-1} E_{z} \xi_{i}=1(i=1,2)$. Здесь и ниже подразумевается предельный переход $n \rightarrow \infty$ такой, что $0<c \leqslant \lim \inf c_{n} \leqslant \lim \sup c_{n} \leqslant$ $C<\infty$, где $c_{n}:=n_{2} / n_{1}$. Полагая $z_{i}=\exp \left(-\delta_{i} n_{i}^{-1 / 3}\right)$, находим $\delta_{1}=\left(6 \rho_{1} / \pi^{2}\right)^{1 / 3} c_{n}^{1 / 3}$, $\delta_{2}=\delta_{1} c_{n}^{-2 / 3}$, где $\rho_{1}:=\int_{0}^{\infty} t \ln G\left(e^{-t}\right) d t$.

3. Основные результаты. Удобно перейти к параметризации, связанной с наклоном звеньев ломаной. Положим $X(s):=\left\{x \in X: x_{2} / x_{1} \leqslant s c_{n}\right\}, s \in[0, \infty]$, и обозначим через $\xi_{i}(s):=\sum_{x \in X(s)} x_{i} \nu(x)(i=1,2)$ координаты “текущей” вершины ломаной $\Gamma$.

Tеорема 1. Обозначим $u_{1}(s):=1-(1+s)^{-2}, u_{2}(s):=s^{2}(1+s)^{-2}$. Тогда при каждом $s \in[0, \infty]$ имеем $\lim _{n \rightarrow \infty} E\left[n_{i}^{-1} \xi_{i}(s)\right]=u_{i}(s)(i=1,2)$, и для любого $\varepsilon>0$

$$
\lim _{n \rightarrow \infty} P_{n}\left\{\sup _{0 \leqslant s \leqslant \infty}\left|n_{i}^{-1} \xi_{i}(s)-u_{i}(s)\right| \leqslant \varepsilon\right\}=1, \quad i=1,2 .
$$


Заметим, что функции $u_{1}(s), u_{2}(s)$ параметрически задают кривую $\gamma_{0}$ (см. п. 1). Пусть $\widetilde{\Gamma}_{n}-$ ломаная, получающаяся из $Г$ при сжатии $\left(x_{1}, x_{2}\right) \mapsto\left(x_{1} / n_{1}, x_{2} / n_{2}\right)$. Из теоремы 1 вытекает

СледСтвиЕ. При всех $\varepsilon>0$ справедливо равенство $\lim _{n \rightarrow \infty} P_{n}\left\{d\left(\widetilde{\Gamma}_{n}, \gamma_{0}\right) \leqslant \varepsilon\right\}=1$, где $d(\cdot, \cdot)$ - метрика Хаусдорфа на множестве компактов в $\mathbb{R}^{2}$.

ЗАмечАниЕ. Согласно следствию из теоремы 1 предельная кривая $\gamma_{0}$ оказывается одинаковой для всех распределений $P_{n}$ из рассматриваемого класса. Это подтверждает высказанную Ю.В. Прохоровым гипотезу о возможной устойчивости предельной формы выпуклых ломаных.

Пусть $\eta_{n}(s), s \in[0, \infty],-$ двумерный случайный процесс с компонентами

$$
\eta_{1 n}(s):=c_{n}^{1 / 6} n_{1}^{-2 / 3}\left[\xi_{1}(s)-E_{z} \xi_{1}(s)\right], \quad \eta_{2 n}(s):=c_{n}^{-1 / 6} n_{2}^{-2 / 3}\left[\xi_{2}(s)-E_{z} \xi_{2}(s)\right] .
$$

Имеет место следующая центральная предельная теорема.

Теорема 2. В смысле сходимости конечномерных распределений относительно $P_{n}$ прочесс $\eta_{n}(s)$ сходится при $n \rightarrow \infty \kappa$ двумерному гауссовскому процессу $\eta^{0}(s)$ с нулевым средним и ковариационной матрицей $\operatorname{Cov}\left(\eta^{0}(s), \eta^{0}(s+h)\right)=B(s)-B(u) B^{-1} B(s+h)$, где $B(s)$ - матрица с әлементами

$$
B_{i j}(s)=6\left(\frac{\pi^{2}}{6 \rho_{1}}\right)^{1 / 3} \int_{0}^{s} \frac{\tau^{i+j-2} d \tau}{(1+\tau)^{4}}, \quad i, j \in\{1,2\} .
$$

Перейдем к изучению статистики количества целых точек и количества вершин на ломаной $Г$. Рассмотрим соответствующие случайные процессы

$$
\chi_{\Gamma}(s):=\sum_{x \in X(s)} \nu(x) \text { и } \vartheta_{\Gamma}(s):=\sum_{x \in X(s)} I\{\nu(x)>0\}, \quad s \in[0, \infty] .
$$

Напомним, что $\rho_{1}$ определено в конце п. 2 , и обозначим

$$
\rho_{0}:=\int_{0}^{\infty} \ln G\left(e^{-t}\right) d t, \quad \sigma_{1}:=\int_{0}^{\infty} t\left(1-1 / G\left(e^{-t}\right)\right) d t .
$$

Tеорема 3. Для любого $\varepsilon>0$

$$
\begin{aligned}
& \lim _{n \rightarrow \infty} P_{n}\left\{\sup _{0 \leqslant s \leqslant \infty}\left|\frac{\chi_{\Gamma}(s)}{\left(n_{1} n_{2}\right)^{1 / 3}}-\rho_{0}\left(\frac{\rho_{1}^{2} \pi^{2}}{6}\right)^{-1 / 3} \frac{s}{1+s}\right| \leqslant \varepsilon\right\}=1, \\
& \lim _{n \rightarrow \infty} P_{n}\left\{\sup _{0 \leqslant s \leqslant \infty}\left|\frac{\vartheta_{\Gamma}(s)}{\left(n_{1} n_{2}\right)^{1 / 3}}-\sigma_{1}\left(\frac{\rho_{1}^{2} \pi^{2}}{6}\right)^{-1 / 3} \frac{s}{1+s}\right| \leqslant \varepsilon\right\}=1 .
\end{aligned}
$$

ЗАмечАниЕ. Теорема 3 показывает, что предельная доля целых точек, приходящихся на одно звено, равна $\rho_{0} / \sigma_{1}$. В случае, когда распределение $P_{n}$ индуцировано мерой $Q_{z}^{(r)}$ (см. п. 2), эта доля возрастает по $r$. При $r=1$ (равномерное распределение на $\mathscr{L}_{n}$ ) указанное отношение равно $\pi^{2} / 6$, что совпадает с плотностью множества $X$ пар взаимно простых целых чисел среди всех точек $\mathbb{Z}_{+}^{2}$. Отметим еще, что предельные функции, фигурирующие в теореме 3 , становятся линейными в параметризации $\varkappa=s /(1+s)$, отвечающей переходу к аффинной кривизне для кривой $\gamma_{0}(\mathrm{cp} .[1])$.

Авторы выражают благодарность Я. Г. Синаю за внимание к работе и А. В. Булинскому за полезные замечания.

\section{СПИСОК ЛИТЕРАТУРЫ}

[1] Вершик А. М. // Функц. анализ и его прил. 1994. Т. 28. № 1. С. 16-25. [2] Bárány I. // Discrete Comput. Geom. 1995. V. 13. P. 279-295. [3] Синай Я. Г. // Функц. анализ и его прил. 1994. Т. 28. № 2. С. 41-48. [4] Феллер В. Введение в теорию вероятностей и ее приложения. Т. 1. М.: Мир, 1984.

Московский государственный университет им. М. В. Ломоносова; Международньй институт теории прогноза землетрясений и математической геофизики РАН

E-mail: lvb@mech.math.msu.su, sakhavet@mitp.ru
Принято редколлегией 21.07.1999 